\title{
Relações entre os atributos de qualidade de atenção aos usuários hipertensos e diabéticos na Estratégia Saúde da Família e o controle dos fatores prognósticos de complicações
}

\author{
Relationships between quality of care characteristics \\ directed to hypertensive and diabetic patients of the Family Health \\ Strategy and the control of prognostic factors for complications
}

\author{
Annick Fontbonne ${ }^{1}$, Elisabete Costa de Souza², Janine Christina Nunes de Oliveira², \\ Heloísa de Melo Rodrigues ${ }^{3}$, Wayner Vieira de Souza², \\ Eduarda Ângela Pessoa Cesse ${ }^{2}$
}

\begin{abstract}
Resumo
Introdução: Apesar de a Estratégia Saúde da Família (ESF) ser concebida para atender aos requisitos da atenção a portadores de agravos crônicos, verifica-se na prática que o controle da hipertensão arterial sistêmica (HAS) e do diabetes mellitus (DM) continua insatisfatório. Objetivo: Objetivou-se investigar possíveis influências da qualidade da atenção sobre o controle dos fatores prognósticos de complicações da HAS e do DM em usuários cadastrados na ESF em Pernambuco. Método: Estudo transversal em uma amostra aleatória de 1.607 hipertensos e diabéticos cadastrados na ESF em 35 municípios do Estado de Pernambuco. A qualidade da atenção foi avaliada por "acessibilidade", "serviços disponíveis", "serviços prestados" e "satisfação". Relações com o controle dos fatores prognósticos foram verificadas pelo teste do qui-quadrado. Resultados: A prática de atividade física foi o desfecho que mais se encontrou relacionado com a qualidade da atenção; ao contrário, qualidade da atenção não se mostrou associada à cessação do tabagismo. Relações entre qualidade da atenção e controle do peso, da pressão arterial ou do diabetes foram inexistentes ou paradoxais. Conclusão: Atenção de boa qualidade pode melhorar a adesão dos usuários à prática de atividade física.
\end{abstract}

Palavras-chave: Estratégia Saúde da Família; qualidade da assistência à saúde; prevenção \& controle; hipertensão; diabetes mellitus.

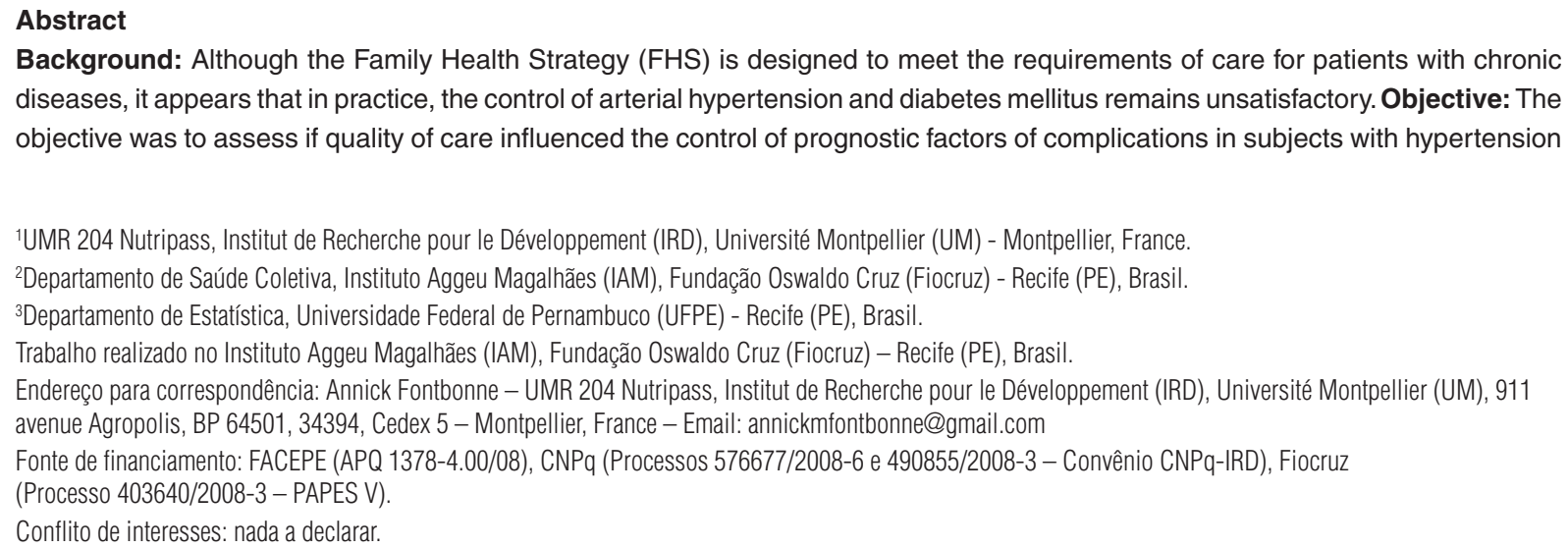

Background: Although the Family Health Strategy (FHS) is designed to meet the requirements of care for patients with chronic diseases, it appears that in practice, the control of arterial hypertension and diabetes mellitus remains unsatisfactory. Objective: The objective was to assess if quality of care influenced the control of prognostic factors of complications in subjects with hypertension 
or diabetes enrolled in the FHS in Pernambuco. Method: This is a cross-sectional study on a random sample of 1607 hypertensive and diabetic subjects enrolled with the FHS in 35 municipalities in the state of Pernambuco. Quality of care was evaluated by "accessibility", "services available", "services provided" and "satisfaction". The relationships with control of prognostic factors were sought using the chi-square test. Results: The practice of physical activity was the outcome that was most closely linked to quality of care; on the other hand, quality of care was not found associated with smoking cessation. The relationships between quality of care and control of weight, blood pressure or diabetes were nonexistent or paradoxical. Conclusion: Good quality of care may have a positive impact on the practice of physical activity by hypertensive or diabetic subjects.

Keywords: Family Health Strategy; quality of health care; prevention \& control; hypertension; diabetes mellitus.

\section{INTRODUÇÃO}

As doenças crônicas não transmissíveis constituem um dos maiores problemas de saúde pública na atualidade ${ }^{1}$. Entre elas, destacam-se a hipertensão arterial sistêmica (HAS) e o diabetes mellitus (DM) tipo 2. Segundo dados da Pesquisa Nacional de Saúde de 2013, 21\% dos indivíduos com 18 anos ou mais apresentaram diagnóstico prévio de HAS, e 6,2\%, de DM, o que corresponde a aproximadamente 31,3 milhões de pessoas com HAS e 9,1 milhões com $\mathrm{DM}^{2}$. Esses agravos, quando não controlados, geram uma série de complicações, como doenças cardiovasculares e renais, e, mais especificamente, no caso do DM, complicações oculares e neurais, que podem conduzir à cegueira e a amputações, além de ter altos custos para o indivíduo e a sociedade ${ }^{3}$.

A prevenção e o manejo da HAS e do DM são ações programáticas prioritárias dos serviços de atenção primária à saúde (APS), não somente em razão da sua alta prevalência e responsabilidade nas grandes causas de morbimortalidade no país, mas também por serem consideradas condições sensíveis a esse nível de atenção, havendo evidência acumulada de que o bom manejo desses problemas, na APS, evita hospitalizações e mortes por complicações cardiovasculares e cerebrovasculares ${ }^{4}$. A Estratégia Saúde da Família (ESF), organização da APS mais difundida hoje no Brasil, está teoricamente concebida de maneira a atender aos requisitos da atenção a portadores de agravos crônicos, como HAS e DM: equipe multiprofissional de proximidade, encarregada da educação e promoção da saúde, com modo de agir proativo, organizadora de uma rede de atenção com foco no usuário ${ }^{5}$.

No entanto, verifica-se na prática dos serviços que o controle da HAS e do DM continua insatisfatório. Em um artigo anterior baseado no estudo SERVIDIAH, que incluiu uma amostra representativa de hipertensos e diabéticos cadastrados nas ESF de Pernambuco, observou-se que menos da metade dos hipertensos tinha pressão arterial controlada e que somente $30 \%$ dos diabéticos apresentavam hemoglobina glicada (indicadora do controle glicêmico) abaixo do nível recomendado de 7\%; ademais, apesar de cerca de $75 \%$ dos entrevistados apresentarem excesso de peso, somente $16 \%$ dos hipertensos e $14 \%$ dos diabéticos disseram seguir uma dieta para perder peso, enquanto $70 \%$ afirmaram não praticar nenhuma atividade física ${ }^{6}$.
Com o intuito de questionar se esses resultados podiam ser melhorados com aprimoramento da qualidade da atenção, analisou-se, a partir do estudo SERVIDIAH, a relação entre os indicadores de qualidade de atenção e os principais fatores prognósticos de complicações cardiovasculares em usuários com HAS ou DM.

\section{MÉTODO}

\section{O estudo SERVIDIAH}

A presente análise foi realizada com os dados do estudo SERVIDIAH (Avaliação de SERVIços de atenção à saúde para DIAbéticos e Hipertensos no âmbito da Estratégia Saúde da Família), um estudo epidemiológico transversal conduzido em 2010 em uma amostra representativa de usuários hipertensos e diabéticos tipo 2, com mais de 20 anos de idade, cadastrados na Estratégia Saúde de Família do Estado de Pernambuco. Esse estudo abrangeu 35 municípios, dos quais 16 de pequeno porte ( $<20$ mil habitantes), 16 de médio porte (20 mil a 100 mil habitantes) e 3 de grande porte (> 100 mil habitantes), incluindo a capital do Estado, Recife. Em um segundo estágio, foram sorteadas 207 equipes da ESF atuantes nesses municípios. Em cada equipe da ESF, foi realizado um sorteio sistemático entre 3 e 6 usuários com hipertensão (sem diabetes) e entre 3 e 6 usuários com diabetes (com ou sem hipertensão associada), utilizando o cadastro manual dos agentes comunitários de saúde. A amostra final foi composta de 1.607 usuários.

Os dados foram coletados por meio da aplicação de questionário estruturado por pesquisadores de campo previamente treinados para as entrevistas. Incluiu-se um vasto leque de perguntas, focando na história da condição crônica e seu acompanhamento pela ESF, mas contemplando também indicadores socioeconômicos, estilo de vida, gastos com os cuidados, complicações etc. Foram realizadas medidas antropométricas (peso, altura, circunferências da cintura e dos quadris), aferição da pressão arterial e, para os usuários diabéticos, dosagem da hemoglobina glicada (HbA1c) em uma amostra de sangue capilar, por aparelho portátil, dando o resultado dentro de alguns minutos (in2it ${ }^{\oplus}$ da Bio-Rad).

Os usuários foram entrevistados em seus domicílios ou em uma sala do posto da equipe da ESF, após terem sido informados sobre os objetivos e os procedimentos da pesquisa e assinado o Termo de Consentimento Livre e Esclarecido. O estudo SERVIDIAH obteve aprovação do Comitê de Ética em Pesquisa 
do Centro de Pesquisas Aggeu Magalhães (CEP/CpqAM) (Registro $n^{\circ}$ 43/2008) e da Comissão Nacional de Ética em Pesquisa (CONEP) (Parecer no 889/2008).

Mais detalhes sobre a metodologia do estudo SERVIDIAH podem ser encontrados em Fontbonne et $\mathrm{al}^{6}$.

\section{Variáveis da análise}

Para a presente análise, foram extraídas do questionário do estudo SERVIDIAH as variáveis indicadoras da qualidade da atenção recebida pelo usuário com hipertensão e/ou diabetes, de um lado, e as variáveis indicadoras do controle dos fatores prognósticos de complicações da hipertensão e do diabetes, de outro lado.

A qualidade da atenção foi verificada no questionário do estudo SERVIDIAH, utilizando os quesitos "acessibilidade" e "integralidade" do PCATool brasileiro para adultos ${ }^{7}$, além do quesito "satisfação", acrescentado por Harzheim et al. ${ }^{8}$ para avaliar a qualidade dos serviços de atenção primária à saúde em Porto Alegre. A acessibilidade incluía três perguntas que tratavam sobre: agendamento das consultas (sim/não), facilidade em marcar hora (fácil/difícil) e tempo de espera na unidade de saúde antes da consulta (menos/mais de 30 minutos). A integralidade dizia respeito aos serviços disponíveis e aos serviços prestados. Serviços disponíveis foram avaliados por meio das seguintes questões: se algum profissional da ESF tinha indagado sobre a prática de atividade física ( $\mathrm{sim} / \mathrm{não})$, o hábito de fumar ( $\operatorname{sim} /$ não) e o consumo de álcool (sim/não); tinha incentivado a prática de atividade física ( $\mathrm{sim} / \mathrm{não})$; tinha chamado a atenção do usuário sobre o fato de ele ter que perder peso (sim/não). Serviços prestados foram avaliados em dois blocos: as medições realizadas nos últimos 12 meses (peso, altura, circunferência da cintura e circunferência dos quadris: sim/não) e os exames complementares solicitados nos últimos 12 meses (sumário de urina, dosagem do colesterol e eletrocardiograma: sim/não). O quesito "satisfação" incluía 11 perguntas, cujas respostas, originalmente classificadas em quatro níveis (muito satisfeito, satisfeito, pouco satisfeito e não satisfeito), foram reclassificadas em "satisfeito" (muito satisfeito ou satisfeito) e "insatisfeito" (pouco satisfeito ou não satisfeito). Esse quesito explorava a satisfação com: o atendimento por parte da recepção; a cordialidade do profissional; a atenção dada pelo profissional; a realização do exame clínico; o desenrolar da consulta de modo geral; as explicações sobre a doença; as explicações sobre o prognóstico da doença; as explicações sobre o uso dos remédios; o fornecimento dos remédios; a confiança despertada pelo profissional e a confiança na receita.

Os indicadores do controle dos fatores prognósticos de complicações eram os seguintes:

1. Controle da pressão arterial: controlado se a pressão arterial sistólica estivesse abaixo de $140 \mathrm{mmHg}$ (hipertensos sem diabetes) ou $130 \mathrm{mmHg}$ (diabéticos com ou sem hipertensão) e a pressão arterial diastólica estivesse abaixo de $90 \mathrm{mmHg}$ (hipertensos sem diabetes) ou $80 \mathrm{mmHg}$ (diabéticos com ou sem hipertensão) ${ }^{9}$;

2. Controle do diabetes (somente para usuários diabéticos): controlado se a HbAlc estivesse abaixo de $7 \%{ }^{10}$;

3. Índice de massa corporal (IMC), ou seja, peso em quilogramas dividido pelo quadrado da altura em metros, abaixo de 25 $\mathrm{kg} / \mathrm{m}^{2}$, limite acima do qual se define o sobrepeso ${ }^{11}$;

4. Prática de atividade física de lazer, no mínimo leve, com regularidade (sim/não). A atividade física de lazer praticada de forma regular foi autorreferida como "nenhuma", "leve" (por exemplo, caminhar), "moderada" (por exemplo, praticar ginástica) ou "vigorosa" (competição esportiva);

5. Seguimento de uma dieta para perder peso (sim/não);

6. Cessação do hábito de fumar (sim/não).

\section{Análise estatística}

Todas as variáveis retidas para a análise foram codificadas, de maneira dicotômica, com o valor 0 , indicando "não qualidade" ou "não controle", e o valor 1, o oposto. Por exemplo, se a unidade de saúde agendava as consultas (primeiro item da "acessibilidade"), valia 1 ; senão, valia 0 ; se o usuário tinha pressão arterial controlada, valia 1 ; caso contrário, valia 0 .

Para cada quesito que dizia respeito à qualidade de atenção, um escore foi calculado, somando os valores das variáveis constitutivas desse quesito e dividindo pelo número delas. Após estudo das distribuições desses escores, agrupamentos para fins de análise foram realizados: duas categorias para a acessibilidade (escore $=1$, "perfeita"; ou escore $<1$, "imperfeita"); duas categorias para os serviços disponíveis (escore $\geq 0,5$, "bom"; ou escore $<0,5$, "insuficiente"); três categorias para os serviços prestados/medições (escore $=1$, "todas"; escore $>0$ e $<1$, "algumas"; escore $=0$, "nenhuma"); duas categorias para os serviços prestados/exames complementares (escore $>0$, "ao menos um"; ou escore $=0$, "nenhum"); três categorias para a satisfação do usuário (escore $=1$, "muito satisfeito"; escore $<1$ e $\geq 0,6$, "satisfeito"; escore < 0,6, "pouco satisfeito").

As relações entre os quesitos referentes à qualidade da atenção e aos indicadores do controle dos fatores prognósticos de complicações foram estudadas por tabelas cruzadas, e sua significância estatística foi avaliada pelo teste do qui-quadrado, com nível de significância de 0,05 . O software estatístico Stata, versão 13 (StataCorp LP, College Station, TX, USA), foi utilizado para realizar esses cálculos.

\section{RESULTADOS}

\section{Características gerais da amostra}

A amostra era composta de 481 homens e 1.126 mulheres, com idade média de 60,9 anos (desvio-padrão: 13,5 anos). Dos 1.607 usuários entrevistados, 785 tinham hipertensão arterial sem diabetes e 822 eram diabéticos, dos quais $77 \%$ 
tinham hipertensão arterial associada. No que diz respeito aos fatores prognósticos de complicações, notou-se que 34,2\% tinham a pressão arterial controlada; entre os diabéticos, 30,7\% tinham a $\mathrm{HbAlc}$ abaixo de $7 \% ; 25,8 \%$ possuíam IMC abaixo de $25 \mathrm{~kg} / \mathrm{m}^{2}$, indicando peso normal; $30,3 \%$ praticavam atividade física com regularidade; $15,1 \%$ seguiam uma dieta para perder peso; e 41,3\% tinham cessado o hábito de fumar.

Relações entre quesitos de qualidade de atenção e variáveis indicadoras do controle dos fatores prognósticos de complicações

O quesito "acessibilidade" não demonstrou relações significativas com o controle dos fatores prognósticos, a não ser com o controle glicêmico, entre os usuários diabéticos, que eram $32,8 \%$ a ter $\mathrm{HbA1c}$ abaixo de $7 \%$ no caso de acessibilidade "perfeita" (ou seja, consultas eram agendadas, era fácil marcar hora e o tempo de espera antes da consulta não ultrapassava meia hora), comparado com $24,9 \%$ quando a acessibilidade era "imperfeita" $(\mathrm{p}=0,039)$ (Tabela 1).
Mais relações foram evidenciadas para o quesito "serviços disponíveis" (Tabela 2): houve relação positiva com a proporção de usuários praticando atividade física regularmente e seguindo uma dieta para perder peso (respectivamente, 34,7 e 20,6\% em caso de bom nível de "serviços disponíveis", comparado com 25,2 e 8,8\% em serviços com menos bom nível nesse quesito, $\mathrm{p}<0,001$ ); porém, a relação com o IMC foi paradoxal, com uma proporção menor de usuários com peso normal em caso de bom nível de "serviços disponíveis" (20,9\% vs. 32\%,p < 0,001).

Outra relação paradoxal foi evidenciada para o quesito "serviços prestados/medições realizadas nos últimos 12 meses", em que a proporção de usuários com pressão arterial controlada aumentou de 28,8 a $37 \%$ ( $p=0,025)$, enquanto o quesito passava de "todas as medições" a "nenhuma" (Tabela 3). Já a outra relação significativa com a atividade física foi no sentido esperado: tinha uma proporção menor de usuários praticando atividade física regularmente $(24,9 \%)$ quando nenhuma medida tinha sido realizada nos últimos 12 meses, comparada com mais de $32 \%$ quando essas medidas tinham sido total ou parcialmente realizadas $(\mathrm{p}<0,001)$.

Tabela 1. Relação entre o quesito de qualidade de atenção "acessibilidade" e o controle dos fatores prognósticos de complicações de hipertensão ou diabetes. Pernambuco, 2010

\begin{tabular}{lccc} 
& Acessibilidade perfeita & Acessibilidade imperfeita & Valor de p \\
\% com pressão arterial controlada & $32,6 \%$ & $33,3 \%$ & 0,805 \\
\% diabéticos com HbA1c controlada & $32,8 \%$ & $24,9 \%$ & $\mathbf{0 , 0 3 9}$ \\
\% com IMC abaixo de $25 \mathrm{~kg} / \mathrm{m}^{2}$ & $24,7 \%$ & $25,9 \%$ & 0,644 \\
\% com atividade física de rotina & $32,4 \%$ & $31,0 \%$ & 0,639 \\
\% seguindo dieta para perder peso & $17,6 \%$ & $15,1 \%$ & 0,261 \\
\% ex-fumantes & $43,3 \%$ & $39,7 \%$ & 0,217 \\
\hline
\end{tabular}

IMC = índice de massa corporal

Tabela 2. Relação entre o quesito de qualidade de atenção "serviços disponíveis" e o controle dos fatores prognósticos de complicações de hipertensão ou diabetes. Pernambuco, 2010

\begin{tabular}{lccc} 
& Disponibilidade boa & Disponibilidade insuficiente & Valor de p \\
\% com pressão arterial controlada & $35,3 \%$ & $32,7 \%$ & 0,270 \\
\% diabéticos com HbA1c controlada & $32,1 \%$ & $29,3 \%$ & 0,407 \\
\% com IMC abaixo de $25 \mathrm{~kg} / \mathrm{m}^{2}$ & $20,9 \%$ & $32,0 \%$ & $\mathbf{0 , 0 0 1}$ \\
\% com atividade física de rotina & $34,7 \%$ & $25,2 \%$ & $\mathbf{0 , 0 0 1}$ \\
\% seguindo dieta para perder peso & $20,6 \%$ & $8,8 \%$ & $\mathbf{0 , 0 0 1}$ \\
\% ex-fumantes & $39,5 \%$ & $43,3 \%$ & 0,129 \\
\hline
\end{tabular}

IMC = índice de massa corporal

Tabela 3. Relação entre o quesito de qualidade de atenção "medições realizadas nos últimos 12 meses" e o controle dos fatores prognósticos de complicações de hipertensão ou diabetes. Pernambuco, 2010

\begin{tabular}{|c|c|c|c|c|}
\hline & Todas as medições & Algumas das medições & Nenhuma das medições & Valor de $\mathbf{p}$ \\
\hline$\%$ com pressão arterial controlada & $28,8 \%$ & $34,3 \%$ & $37,0 \%$ & 0,025 \\
\hline$\%$ diabéticos com HbA1c controlada & $29,5 \%$ & $30,8 \%$ & $30,9 \%$ & 0,935 \\
\hline$\%$ com IMC abaixo de $25 \mathrm{~kg} / \mathrm{m}^{2}$ & $26,4 \%$ & $25,3 \%$ & $26,0 \%$ & 0,927 \\
\hline$\%$ com atividade física de rotina & $32,3 \%$ & $35,5 \%$ & $24,9 \%$ & 0,001 \\
\hline$\%$ seguindo dieta para perder peso & $14,5 \%$ & $17,1 \%$ & $13,9 \%$ & 0,302 \\
\hline$\%$ ex-fumantes & $42,1 \%$ & $39,4 \%$ & $41,2 \%$ & 0,687 \\
\hline
\end{tabular}

IMC = índice de massa corporal 
Para o quesito "serviços prestados/exames complementares nos últimos 12 meses", evidenciou-se relação somente com a prática de atividade física, mais frequente quando ao menos um dos três exames contemplados (sumário de urina, dosagem do colesterol e eletrocardiograma) tinha sido solicitado: $33,3 \%$ vs. $28,0 \%$, com $\mathrm{p}=0,024$ (Tabela 4 ).
Também no caso do último quesito analisado, a "satisfação", somente a atividade física se mostrou relacionada com proporção de praticantes acompanhando o grau de satisfação com a atenção: $35,9 \%$ entre os usuários muito satisfeitos, 30,3\% entre os satisfeitos e $24 \%$ entre os pouco satisfeitos, com $p<0,001$ (Tabela 5).

Tabela 4. Relação entre o quesito de qualidade de atenção "exames complementares solicitados nos últimos 12 meses" e o controle dos fatores prognósticos de complicações de hipertensão ou diabetes. Pernambuco, 2010

\begin{tabular}{|c|c|c|c|}
\hline & Ao menos um & Nenhum & Valor de p \\
\hline \% com pressão arterial controlada & $34,8 \%$ & $33,7 \%$ & 0,642 \\
\hline \% diabéticos com HbA1c controlada & $27,8 \%$ & $33,1 \%$ & 0,110 \\
\hline$\%$ com IMC abaixo de $25 \mathrm{~kg} / \mathrm{m}^{2}$ & $24,7 \%$ & $26,7 \%$ & 0,393 \\
\hline$\%$ com atividade física de rotina & $33,3 \%$ & $28,0 \%$ & 0,024 \\
\hline$\%$ seguindo dieta para perder peso & $15,5 \%$ & $14,8 \%$ & 0,709 \\
\hline$\%$ ex-fumantes & $43,8 \%$ & $39,3 \%$ & 0,069 \\
\hline
\end{tabular}

$\mathrm{IMC}=$ índice de massa corporal

Tabela 5. Relação entre o quesito de qualidade de atenção "satisfação" e o controle dos fatores prognósticos de complicações de hipertensão ou diabetes. Pernambuco, 2010

\begin{tabular}{|c|c|c|c|c|}
\hline & Muito satisfeito & Satisfeito & Pouco satisfeito & Valor de p \\
\hline$\%$ com pressão arterial controlada & $33,5 \%$ & $32,4 \%$ & $34,8 \%$ & 0,718 \\
\hline$\%$ diabéticos com HbAlc controlada & $29,8 \%$ & $30,2 \%$ & $32,2 \%$ & 0,851 \\
\hline$\%$ com IMC abaixo de $25 \mathrm{~kg} / \mathrm{m}^{2}$ & $26,3 \%$ & $25,1 \%$ & $25,7 \%$ & 0,909 \\
\hline$\%$ com atividade física de rotina & $35,9 \%$ & $30,3 \%$ & $24,0 \%$ & 0,001 \\
\hline$\%$ seguindo dieta para perder peso & $15,6 \%$ & $15,5 \%$ & $13,8 \%$ & 0,705 \\
\hline$\%$ ex-fumantes & $38,5 \%$ & $42,2 \%$ & $42,6 \%$ & 0,340 \\
\hline
\end{tabular}

IMC = índice de massa corporal

\section{DISCUSSÃO}

Dentro da amostra do estudo SERVIDIAH, representativa da população de hipertensos e diabéticos acompanhados pela ESF no Estado de Pernambuco em 2010, poucas relações foram evidenciadas entre os quesitos da qualidade da atenção recebida e os indicadores de controle dos fatores prognósticos de complicações. Vale ressaltar que a qualidade da atenção foi verificada a partir de perguntas dirigidas aos usuários; portanto, podem ser sujeitas à subjetividade do entrevistado e a vieses de memória, o que pode dificultar a detecção de relações com outras variáveis. No entanto, essas dificuldades acompanham toda aplicação de instrumento a usuários dos serviços de saúde e não impediram a validação do instrumento $\mathrm{PCATool}^{7}$, do qual o questionário do estudo SERVIDIAH se inspirou, no que diz respeito aos indicadores da qualidade da atenção. Outra limitação importante é que o questionário do estudo SERVIDIAH não contemplou todos os itens do PCATool, e, por consequência, os quesitos de qualidade da atenção não puderam ser analisados da forma padronizada recomendada no manual de utilização desse instrumento ${ }^{7}$.

Entre as relações evidenciadas, destaca-se a ligação da prática de atividade física com todos os quesitos de qualidade de atenção, fora o primeiro, "acessibilidade". Apesar de a atividade física autorreferida muitas vezes ser superestimada ${ }^{12}$, não há motivo de pensar que essa superestimação seja maior quando a qualidade de atenção é melhor, ou seja, que o resultado decorra de um viés de informação/mensuração. Esse resultado é muito positivo devido à importância da atividade física no controle da pressão e da glicemia e também na prevenção de muitos agravos, desde doenças cardiovasculares até problemas osteoarticulares $^{13-15}$. O fato de essa relação se destacar, entre outros fatores prognósticos comportamentais (seguir uma dieta para perder peso ou parar de fumar), possa talvez ser atribuído ao contexto de forte incentivo geral à atividade física promovido pelas políticas públicas brasileiras, como a iniciativa das Academias da Saúde, que ganharam respaldo internaciona ${ }^{16}$. Também alguns trabalhos anteriores, revisados em Nied e Franklin ${ }^{17}$, mostraram que, quando a promoção da atividade física pelo serviço é feita de maneira engajada e repetida, ela surte efeitos em pacientes idosos e/ou com condições crônicas.

Isso pode ser mais difícil de atingir para comportamentos que remetem a importantes modificações no estilo de vida, como mudar de hábitos alimentares ou parar de fumar. "Seguir uma dieta para perder peso" ou simplesmente uma dieta "saudável" 
levanta obstáculos não somente culturais ou de convivialidade (comer de maneira diferente dos outros, da família), mas também, em uma população pobre e com baixo grau de instrução, como é o caso dos usuários da ESF, aqui estudados, obstáculos concretos de preços e falta de conhecimentos diante das opções incentivadas pela indústria agroalimentar ${ }^{18-20}$. Apesar dessas dificuldades, que podem se contrapor à atuação dos serviços de atenção básica, mesmo de boa qualidade, o fato de "seguir uma dieta para perder peso" apareceu relacionado, neste estudo, ao quesito de qualidade de atenção "serviços disponíveis". Vale notar que os elementos constitutivos desse quesito eram indagações e conselhos a respeito do estilo de vida por parte dos profissionais. Pode ser que o interesse assim demonstrado tenha influência direta sobre efetivas mudanças no estilo de vida - no caso, mudanças alimentares - por parte dos usuários. Quanto ao tabagismo, ou melhor, ao fato de ter cessado o hábito de fumar, esse foi o único fator prognóstico de complicações estudado a não apresentar nenhuma relação significativa com os quesitos de qualidade de atenção. Sabidamente, o tabagismo é um dos hábitos mais difíceis de mudar, e, no Brasil, apenas uma minoria dos fumantes que tentam parar de fumar recebe aconselhamento de um profissional de saúde sobre como parar e uma parte menor ainda recebe um acompanhamento adequado para esse $\mathrm{fim}^{21}$. Esse quadro mereceria aprimoramento, particularmente em direção a usuários hipertensos ou diabéticos, que têm alto risco cardiovascular, do qual o tabagismo é um fator agravante de maior importância.

Foram poucas as relações entre a qualidade da atenção e os fatores clínicos prognósticos de complicações; quando existiam, revelaram-se bastante paradoxais. O IMC apareceu relacionado com o quesito "serviços disponíveis", porém no sentido de que tinha menos usuários com peso normal nas equipes da ESF onde a disponibilidade era julgada "boa". Uma explicação pode ser que, como a "boa disponibilidade" incluía ter chamado a atenção do usuário sobre o fato de ele ter que perder peso, isso tenha acontecido menos para usuários com peso normal, ou seja, a relação não deve ser interpretada como "falha" da qualidade de atenção, e sim como relação inversa de causa para efeito (quanto menos usuários de peso normal, mais frequente o fato de chamar a atenção sobre a necessidade de perder peso). Outra relação paradoxal foi o controle da pressão arterial com o quesito "medições realizadas nos últimos 12 meses". O mesmo tipo de explicação pode se aplicar: quanto mais usuários com descontrole da pressão arterial, mais frequentemente se realizam as medidas antropométricas, seja por esse descontrole despertar mais atenção por parte dos profissionais, seja, simplesmente, por esses usuários procurarem o serviço de saúde com mais frequência. Essas incertezas de interpretação entre causa e efeito são consequências da limitação decorrente do desenho transversal do estudo SERVIDIAH. Verificou-se, por último, uma relação entre o bom controle glicêmico, julgado pelo valor da HbA1c, com o quesito "acessibilidade". Esse quesito não apresentou nenhuma outra relação significativa com os fatores prognósticos de complicações, assim como o controle glicêmico não apresentou nenhuma outra relação significativa com os quesitos de qualidade da atenção. Essa quase ausência de relações com fatores clínicos, ou existência de relações paradoxais, encontra-se em outros estudos centrados na avaliação da qualidade da atenção. Gonçalves et al. ${ }^{22}$ (p. 241), em um estudo avaliando a qualidade do serviço por meio do PCATool, mostraram que esta não tem relação com o controle do diabetes e concluíram que "[...] é mais provável de melhorar o processo de cuidado do que os desfechos em DM". Um estudo comparando a qualidade da atenção para diabéticos com hipertensão em centros especializados e na atenção básica no Bahrein, país do Golfo Pérsico, observou que pressão e glicemia eram inadequadamente controladas, e isso sem diferença entre os dois tipos de serviço ${ }^{23}$. Estudos prospectivos têm mostrado resultados melhores. Asch et al. puderam relacionar a qualidade da atenção de 12 serviços médicos comunitários nos Estados Unidos com um melhor controle da pressão arterial de hipertensos ao longo de dois anos ${ }^{24}$. Um estudo-piloto, avaliando os resultados de uma intervenção para implantar o modelo de cuidados crônicos ${ }^{25}$ em um consultório de clínico geral, mostrou resultados positivos sobre vários desfechos, incluindo controle do diabetes e da hipertensão, após um ano de intervenção, mas não tinha grupo de comparação ${ }^{26}$.

Essa análise quantitativa, buscando relações entre quesitos de qualidade de atenção e controle dos fatores prognósticos de complicações da hipertensão e do diabetes, em uma amostra representativa de usuários hipertensos e diabéticos acompanhados pela ESF no Estado de Pernambuco, apontou para a possibilidade de que a atenção de boa qualidade seja suscetível de melhorar a adesão dos usuários à prática de atividade física. Mais esforço deve ser dirigido à obtenção de mudanças nos hábitos alimentares e na cessação do tabagismo.

\section{AGRADECIMENTOS}

Os autores desejam agradecer às instituições que financiaram o estudo SERVIDIAH, aos administradores e profissionais da Estratégia Saúde da Família dos municípios participantes do Estado de Pernambuco, aos estudantes dos cursos de Saúde Pública do Instituto Aggeu Magalhães, Fiocruz-PE, que se envolveram nas atividades de seu Laboratório de Avaliação, Monitoramento e Vigilância em Saúde (LAM-Saúde), responsável pelo estudo, e a todo o pessoal que colaborou na coleta, gestão de dados e análise estatística. 


\section{REFERÊNCIAS}

1. Schmidt MI, Duncan BB, Azevedo e Silva G, Menezes AM, Monteiro CA, Barreto SM, et al. Doenças crônicas não transmissíveis no Brasil: carga e desafios atuais. Lancet. 2011 jun;377(9781):1949-61. http://dx.doi. org/10.1016/S0140-6736(11)60135-9. PMid:21561658.

2. Malta DC, Stopa SR, Szwarcwald CL, Gomes NL, Silva JB Jr, Reis AA. A vigilância e o monitoramento das principais doenças crônicas não transmissíveis no Brasil: Pesquisa Nacional de Saúde, 2013. Rev Bras Epidemiol. 2015 dez;18(Supl 2):3-16. http://dx.doi.org/10.1590/19805497201500060002. PMid:27008599.

3. Dib MW, Riera R, Ferraz MB. Estimated annual cost of arterial hypertension treatment in Brazil. Rev Panam Salud Publica. 2010 fev;27(2):125-31. http:// dx.doi.org/10.1590/S1020-49892010000200006. PMid:20339616.

4. Alfradique ME, Bonolo PF, Dourado I, Lima-Costa MF, Macinko J, Mendonça CS, et al. Internações por condições sensíveis à atenção primária: a construção da lista brasileira como ferramenta para medir o desempenho do sistema de saúde (Projeto ICSAP - Brasil). Cad Saúde Pública. 2009 jun;25(6):1337-49. http://dx.doi.org/10.1590/S0102-311X2009000600016. PMid:19503964

5. Mendes EV. O cuidado das condições crônicas na atenção primária à saúde: o imperativo da consolidação da estratégia da saúde da família. Brasília: Organização Pan-Americana de Saúde; 2012.

6. Fontbonne A, Cesse EA, Sousa IM, Souza WV, Chaves VL, Bezerra AF, et al Risk factor control in hypertensive and diabetic subjects attended by the Family Health Strategy in the State of Pernambuco, Brazil: the SERVIDIAH study. Cad Saúde Pública. 2013 jun;29(6):1195-204. http://dx.doi.org/10.1590/ S0102-311X2013000600016. PMid:23778551.

7. Brasil. Ministério da Saúde. Secretaria de Atenção à Saúde. Departamento de Atenção Básica. Manual do instrumento de avaliação da atenção primária à saúde: Primary Care Assessment Tool PCATool-Brasil [Internet]. Brasília: Ministério da Saúde; 2010 [citado em 2016 ago 11]. (Série A. Normas e Manuais Técnicos). Disponível em: http://www5.ensp.fiocruz.br/biblioteca/ dados/txt_519818998.pdf

8. Harzheim E, Duncan BB, Stein AT, Cunha CR, Gonçalves MR, Trindade TG, et al. Quality and effectiveness of different approaches to primary care delivery in Brazil. BMC Health Serv Res. 2006 dez;6(1):156. http://dx.doi. org/10.1186/1472-6963-6-156. PMid:17147819.

9. Sociedade Brasileira de Cardiologia. Sociedade Brasileira de Hipertensão. Sociedade Brasileira de Nefrologia. VI diretrizes brasileiras de hipertensão. Arq Bras Cardiol. 2010 jul;95(1 Supl):1-51.

10. American Diabetes Association. Standards of medical care in diabetes 2014 Diabetes Care. 2014 jan;37(Supl Supl 1):S14-80. http://dx.doi.org/10.2337/ dc14-S014. PMid:24357209.

11. World Health Organization. Obesity: preventing and managing the global epidemic. Report of a WHO consultation. World Health Organ Tech Rep Ser. 2000;894:i-xii, 1-253. PMid:11234459.

12. Tully MA, Panter J, Ogilvie D. Individual characteristics associated with mismatches between self-reported and accelerometer-measured physical activity. PLoS One. 2014 jun;9(6):e99636. http://dx.doi.org/10.1371/journal. pone.0099636. PMid:24919185.

13. Barroso WK, Jardim PC, Vitorino PV, Bittencourt A, Miquetichuc F. Influência da atividade física programada na pressão arterial de idosos hipertensos sob tratamento não-farmacológico. Rev Assoc Med Bras. 2008 jul-ago;54(4):328-33. http://dx.doi.org/10.1590/S0104-42302008000400018. PMid:18719791.

14. Molena-Fernandes CA, Nardo N Jr, Tasca RS, Pelloso SM, Cuman RKN. A importância da associação de dieta e de atividade física na prevenção e controle do Diabetes mellitus tipo 2. Acta Sci Health Sci. 2005;27(2):195205.

15. Coelho CF, Burini RC. Atividade física para prevenção e tratamento das doenças crônicas não transmissíveis e da incapacidade funcional. Rev Nutr Campinas. 2009 nov-dez;22(6):937-46. http://dx.doi.org/10.1590/ S1415-52732009000600015.

16. Simões EJ, Hallal P, Pratt M, Ramos L, Munk M, Damascena W, et al. Effects of a community-based, professionally supervised intervention on physical activity levels among residents of Recife, Brazil. Am J Public Health. 2009 jan;99(1):68-75. http://dx.doi.org/10.2105/AJPH.2008.141978. PMid:19008499.

17. Nied RJ, Franklin B. Promoting and prescribing exercise for the elderly. Am Fam Physician. 2002 fev;65(3):419-26. PMid:11858624.

18. Drewnowski A, Specter SE. Poverty and obesity: the role of energy density and energy costs. Am J Clin Nutr. 2004 jan;79(1):6-16. http://dx.doi. org/10.1093/ajen/79.1.6. PMid:14684391.

19. Claro RM, Monteiro CA. Renda familiar, preço de alimentos e aquisição domiciliar de frutas e hortaliças no Brasil. Rev Saúde Pública. 2010 dez;44(6):1014-20. http://dx.doi.org/10.1590/S0034-89102010000600005. PMid:21107500.

20. Brasil. Ministério da Saúde. Secretaria de Atenção à Saúde. Departamento de Atenção Básica. Guia alimentar para a população brasileira. 2. ed. Brasília: Ministério da Saúde; 2014.

21. Brasil. Ministério da Saúde. Secretaria de Atenção à Saúde. Departamento de Atenção Básica. Estratégias para o cuidado da pessoa com doença crônica: o cuidado da pessoa tabagista. Brasília: Ministério da Saúde; 2015.

22. Gonçalves MR, Harzheim E, Zils AA, Duncan BB. A qualidade da atenção primária e o manejo do diabetes mellitus. Rev Bras Med Fam Comunidade. 2013 out-dez;8(29):235-43. http://dx.doi.org/10.5712/rbmfc8(29)814.

23. Al Khaja KA, Sequeira RP, Damanhori AH. Comparison of the quality of diabetes care in primary care diabetic clinics and general practice clinics. Diabetes Res Clin Pract. 2005 nov;70(2):174-82. http://dx.doi.org/10.1016/j. diabres.2005.03.029. PMid:15890429.

24. Asch SM, McGlynn EA, Hiatt L, Adams J, Hicks J, DeCristofaro A, et al. Quality of care for hypertension in the United States. BMC Cardiovasc Disord. 2005 jan;5(1):1. http://dx.doi.org/10.1186/1471-2261-5-1. PMid:15638933.

25. Coleman K, Austin BT, Brach C, Wagner EH. Evidence on the Chronic Care Model in the new millennium. Health Aff (Millwood). 2009 janfev;28(1):75-85. http://dx.doi.org/10.1377/hlthaff.28.1.75. PMid:19124857.

26. Siminerio LM, Piatt G, Zgibor JC. Implementing the chronic care model for improvements in diabetes care and education in a rural primary care practice. Diabetes Educ. 2005 mar-abr;31(2):225-34. http://dx.doi. org/10.1177/0145721705275325. PMid:15797851.

Recebido em: Jul. 27, 2017 Aprovado em: Ago.13, 2018 